\title{
Matrix metalloproteinase activity in the lung is increased in Hermansky-Pudlak syndrome
}

\author{
Ross Summer ${ }^{1}$, Rachana Krishna', DeLeila Schriner ${ }^{1}$, Karina Cuevas-Mora ${ }^{1}$, Dominic Sales ${ }^{1}$, Rachel Para ${ }^{1}$, \\ Jesse Roman ${ }^{1}$, Carl Nieweld ${ }^{1}$, Bernadette R. Gochuico ${ }^{2}$ and Freddy Romero ${ }^{1,3^{*}}$ [D
}

\begin{abstract}
Background: Hermansky-Pudlak syndrome (HPS) is a rare autosomal recessive disorder characterized by oculocutaneous albinism and platelet dysfunction and can sometimes lead to a highly aggressive form of pulmonary fibrosis that mimics the fatal lung condition called idiopathic pulmonary fibrosis (IPF). Although the activities of various matrix metalloproteinases (MMPs) are known to be dysregulated in IPF, it remains to be determined whether similar changes in these enzymes can be detected in HPS.

Results: Here, we show that transcript and protein levels as well as enzymatic activities of MMP-2 and -9 are markedly increased in the lungs of mice carrying the HPS Ap3b1 gene mutation. Moreover, immunohistochemical staining localized this increase in MMP expression to the distal pulmonary epithelium, and shRNA knockdown of the Ap3b1 gene in cultured lung epithelial cells resulted in a similar upregulation in MMP-2 and -9 expression. Mechanistically, we found that upregulation in MMP expression associated with increased activity of the serine/ threonine kinase Akt, and pharmacological inhibition of this enzyme resulted in a dramatic suppression of MMP expression in Ap3b1 deficient lung epithelial cells. Similarly, levels and activity of different MMPs were also found to be increased in the lungs of mice carrying the Bloc3 HPS gene mutation and in the bronchoalveolar lavage fluid of subjects with HPS. However, an association between MMP activity and disease severity was not detected in these individuals.
\end{abstract}

Conclusions: In summary, our findings indicate that MMP activity is dysregulated in the HPS lung, suggesting a role for these proteases as biological markers or pathogenic players in HPS lung disease.

Keywords: Hermansky-Pudlak syndrome, Matrix metalloproteinase, Pulmonary fibrosis

\section{Background}

Hermansky-Pudlak syndrome (HPS) represents a heterogeneous group of rare autosomal recessive disorders characterized by oculocutaneous albinism, platelet dysfunction and, in some cases, pulmonary fibrosis $[1,2]$. To date, ten different HPS genes have been identified, and each encodes for different proteins involved in the biogenesis or trafficking of lysosomes or lysosome-

\footnotetext{
* Correspondence: Freddy.Romero@jefferson.edu

${ }^{1}$ Center for Translational Medicine and Jane and Leonard Korman Lung

Center, Thomas Jefferson University, Philadelphia, USA

${ }^{3}$ Center for Translational Medicine and Jane and Leonard Korman Respiratory

Institute, Thomas Jefferson University, 1020 Locust Street, JAH 354,

Philadelphia, PA 19107, USA

Full list of author information is available at the end of the article
}

related organelles [2-5]. Although each HPS mutation has been shown to affect pigment and platelet functions, only those genes associated with the HPS-1, HPS-2, or HPS-4 genetic subtypes are linked to pulmonary fibrosis $[1,4]$. Importantly, in these individuals the development of pulmonary fibrosis is often a fatal complication, leading to death within just several years of its initial detection.

Matrix metalloproteinases (MMPs) are a family of zinc-dependent proteolytic enzymes that are best known for their role in degrading extracellular matrix proteins, although are also responsible for activating or inhibiting a wide range of other effector molecules [6, 7]. Dysregulated MMP activity has been linked to the pathogenesis

(c) The Author(s). 2019 Open Access This article is distributed under the terms of the Creative Commons Attribution 4.0 International License (http://creativecommons.org/licenses/by/4.0/), which permits unrestricted use, distribution, and reproduction in any medium, provided you give appropriate credit to the original author(s) and the source, provide a link to the Creative Commons license, and indicate if changes were made. The Creative Commons Public Domain Dedication waiver (http://creativecommons.org/publicdomain/zero/1.0/) applies to the data made available in this article, unless otherwise stated. 
of numerous chronic lung diseases, including asthma, emphysema, cystic fibrosis, and fibrotic lung diseases such as idiopathic pulmonary fibrosis (IPF) [7-11]. Although it remains unclear how alterations in this group of enzymes can lead to such diverse lung pathologies, it has been suggested that differences in the expression of enzymatic subtypes might play a contributory role [7-11]. Indeed, levels of specific MMPs have been shown to be elevated in the blood and bronchoalvelolar lavage fluid (BALF) of patients with IPF [10-13], including MMP-2, MMP-7 and MMP-9. While these changes were originally thought to be important for limiting the severity of fibrotic remodeling (by degrading extracellular matrix proteins), targeted deletion of individual MMP genes in mice has yielded mixed results $[10,14-16]$, emphasizing the complex biology of MMPs in the lung.

MMP activity is under strict regulation by a variety of mechanisms at the transcriptional and post-translational levels. Recent work in several model systems has demonstrated the importance of the phosphatidylinositol 3-kinase (PI3K)-Akt pathway in the regulation of MMPs [17-19]. Akt is a serine/threonine kinase, which controls a wide range of biological processes typified in fibrotic tissues, including those intimately involved in growth, proliferation, migration, and metabolic reprogramming of fibroblasts [20, 21]. Additionally, Akt activity has also been shown to be upregulated in type II alveolar epithelial cells (AEC2) of patients with IPF, and inhibition of enzymatic activity in these cells has proven to be effective in reducing tissue remodeling to bleomycin in the mouse lung [21].

In this study, we employed in vitro and in vivo model systems as well as utilized mouse and human tissues to establish whether levels or activities of MMPs were altered in the HPS lung and to determine whether these changes occurred before or after the onset of pulmonary fibrosis.

\section{Methods}

\section{Animals}

Wild-type, HPS1, and HPS2 mice (C57B/6 J, 8-10 weeks old) were purchased from the Jackson Laboratory (Bar Harbor, ME) and housed in a pathogen-free animal facility at Thomas Jefferson University. HPS1 mice have homozygous mutation of the Hps1 gene, which encodes for a protein called BLOC-3, and HPS2 mice have homozygous mutation in the adaptor protein $3 \mathrm{~b} 1$ (Ap3b1) gene, which is a subunit of the AP-3 protein complex. In general, HPS mice are phenotypically normal, except for a light coat appearance. HPS 1 and 2 mice also have large lamellar bodies in the alveolar epithelial type II cells of their lungs. Both strains of mice are also exquisitely sensitive to bleomycin. Throughout the study period, wild-type and HPS mice were maintained on a standard chow diet
(13.5\% calories from fat, $58 \%$ from carbohydrates, and 28.5\% from protein) and permitted to feed ad libitum. Prior to the initiation of any study, the Institutional Animal Care and Use Committee at Thomas Jefferson University approved all animal protocols.

\section{Human subjects}

The diagnosis of HPS was established based on published criteria [22, 23]. Healthy controls were individuals without any known lung disease. Age, gender and smoking history for subjects are listed in Additional file 1: Table S1. All patients provided written informed consent to protocols 95-HG-0193 (clinicaltrials.gov NCT00001456) and 04-HG-0211 (clinicaltrials.gov NCT00084305). All study protocols were approved by the Institutional Review Boards at Thomas Jefferson University and the National Human Genome Research Institute prior to the initiation of any studies. BAL was performed and samples were processed as previously described [24].

\section{Bleomycin-induced lung injury}

Lung injury was induced by instilling $0.025 \mathrm{U}$ of bleomycin into the posterior oropharynx of anesthetized mice. Because HPS1 and HPS2 mice are more sensitive to bleomycin, lower doses of bleomycin were required for these investigations $[2,25,26]$.

\section{Measurement of MMP-2 and MMP-9 activity}

The activity of MMP-2 and -9 was assessed by gelatin zymography as previously described $[8,27]$. Protein concentration was determined by Pierce ${ }^{\mathrm{Tw}}$ BCA assay kit (Thermo Scientific, Rockford, IL). Murine and human BALF and lung homogenates were separated by electrophoresis using 10\% SDS-polyacrylamide gels containing $0.1 \%$ gelatin. Gels were then washed in $2.5 \%$ triton 100 renaturing buffer followed by overnight incubation in developing buffer. To visualize bands gels were stained with $0.5 \%$ Coomassie blue for $1 \mathrm{~h}$ and then destained with $40 \%$ methanol $10 \%$ acetic acid until clear bands were visualized. Densitometry was performed as previously described and the MMPs activity were normalized for total BALF and lung homogenates protein concentration.

\section{Lung histology}

Lungs were removed en bloc and immersed in fixative at $4{ }^{\circ} \mathrm{C}$ for $18 \mathrm{~h}$. Tissues underwent a series of dehydration steps prior to being embedded in paraffin. Prior to performing immunohistochemical staining we performed antigen retrieval and quenched endogenous peroxidases. Primary antibodies to MMP-2 (Abcam, Cambridge, UK) and MMP-9 (Thermo Scientific, Rockford, IL) were used in our studies. To visualize antibody binding, sections were exposed to Vectastain ABC (Vector Laboratories, Burlingame, CA) followed by the addition of 3,39- 
diaminobenzidine. For negative control slides, the primary antibody was replaced by rabbit IgG, polyclonalisotype control (Abcam, Cambridge, UK).

\section{RNA isolation and analysis}

Gene transcript levels were quantified by real-time PCR as previously described [28]. In brief, RNA was isolated using RNeasy Mini-Kit (QIAGEN, Valencia, CA). All reactions were performed with $1 \mu \mathrm{M}$ of forward and reverse primers along with SYBR Green I GoTaq qPCR Master Mix (Promega, Madison, WI). Primer sets were amplified using protocols previously described [28-30]. All values were normalized to a control gene such as $18 S$.

\section{Cell culture and reagents}

Murine lung epithelial 12 (MLE12) cells were obtained from ATCC (Manassas, VA) and cultured as previously described $[28,29]$. Cells were plated in 6-well plates with or without bleomycin $(50 \mu \mathrm{g} / \mathrm{ml})$ or Akt inhibitor $(1 \mu \mathrm{M})$ - After $24 \mathrm{~h}$, supernatant was collected and centrifuged to remove cellular debris and then stored at $-80^{\circ} \mathrm{C}$. Whole cell lysates were also collected to measure transcript or protein levels.
Lentiviral shRNA generation and transduction to MLE12 cells

pLKO.1-based lentiviral Ap3b1 shRNA constructs (RHS4533; clone ID, TRCN0000118642) were used to silence the AP3 gene in MLE12 cells in order to create cells reminiscent to those in the lungs of HPS-2 patients. Scrambled shRNAs were used as a control. Lentiviral transductions for both $A p 3 b 1$ and scrambled control were performed as previously described [28].

\section{Western blot analysis}

Protein concentration was determined by Pierce $^{\mathrm{Tm}}$ BCA assay kit (Thermo Scientific, Rockford, IL). Aliquots of protein lysates were transferred onto nitrocellulose membranes and then blocked with the Odyssey Blocking Buffer (Li-Cor Biosciences, Lincoln, NE) for $1 \mathrm{~h}$ at RT. This step was followed by an incubation step with a specific polyclonal rabbit primary antibody directed against MMP-2, MMP-9, Akt, phosphorylated Akt, or $\beta$ actin (Sigma-Aldrich, St. Louis, MO). Next, membranes were incubated in a solution containing a donkey antirabbit or anti-mouse antibody (Li-Cor Biosciences, Lincoln, $\mathrm{NE}$ ). After three sequential washes with PBS, immunoblots

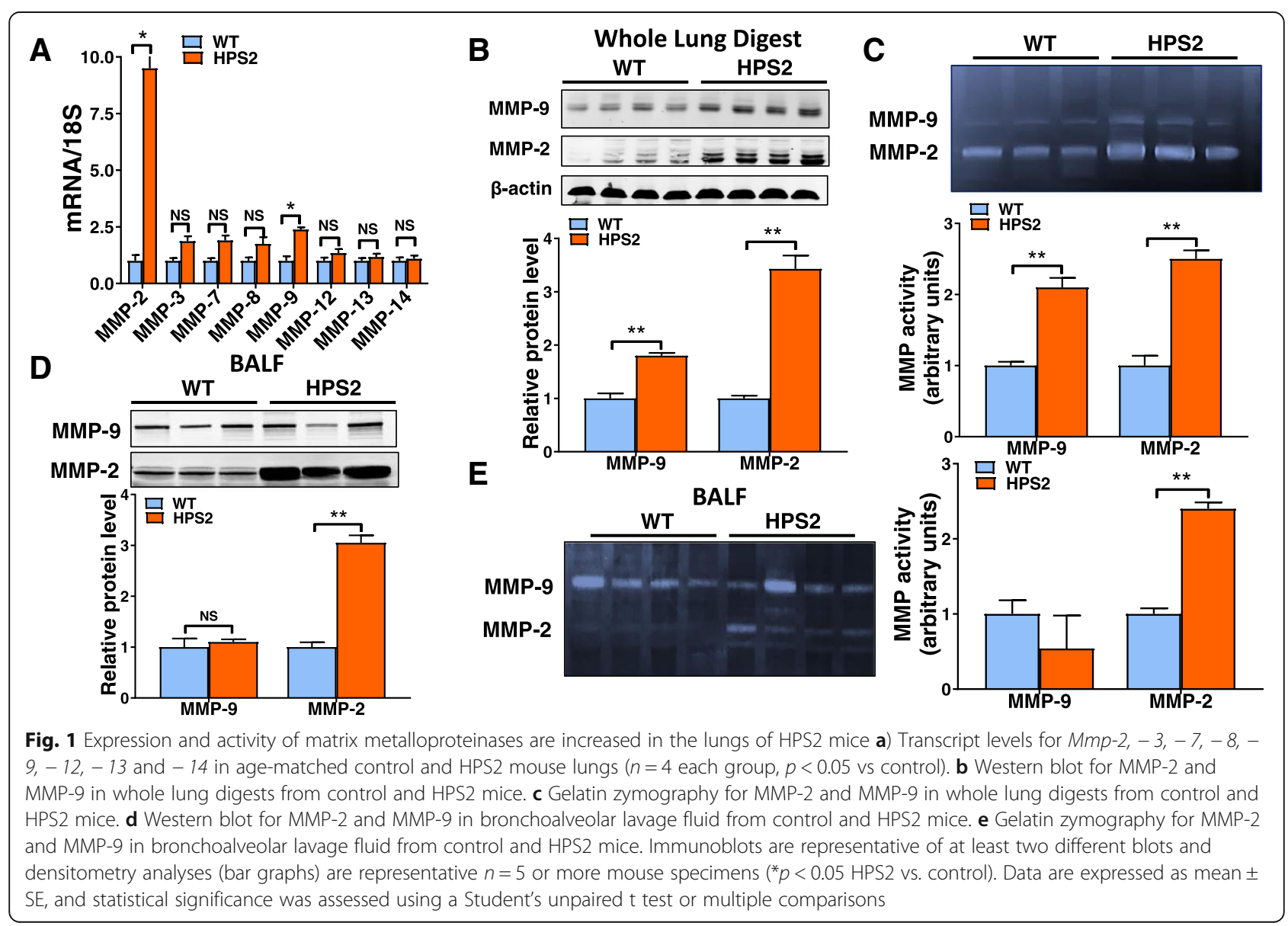


were visualized using the Odyssey infrared imaging system (Li-Cor Biosciences, Lincoln, NE).

\section{Statistical analysis}

Data are expressed as mean + SE. Differences between groups were performed using an unpaired Student's ttest or multiple comparisons with the Bonferroni-Dunn correction. Statistical significance was achieved when $P<0.05$ at $95 \%$ confidence interval.

\section{Results}

Matrix metalloproteinase activity is increased in the lungs of HPS2 mice

To assess whether HPS alters MMP levels in the lung, we first performed quantitative PCR to evaluate transcript levels for several different MMPs known to be expressed in the mouse lung and which have also been linked to lung disease, including $\mathrm{Mmp}-2,-3,-7,-8,-9,-12$ and -14 . As demonstrated in Fig. 1a, we found that transcript levels for each of the Mmps evaluated were readily detectable in the lungs of wild-type mice and that levels for most, if not all, Mmps were upregulated in the lungs of HPS2 mice. However, only transcript levels for Mmp-2 and -9 were found to be significantly increased ( $p$-value $<$ $0.05)$ relative to controls, and only levels of $M m p-2$ and Mmp-9 were increased by more than 2 -fold. Consistent with the marked upregulation in Mmp-2 and Mmp-9 expression, we found that protein levels and enzymatic activity for each of these enzymes were dramatically increased in whole lung tissue digests of HPS2 mice (Fig. 1b, c). In contrast, only levels and enzymatic activity of MMP-2 were increased in BALF (Fig. 1d, e). Altogether, these findings indicate that expression and activity of MMPs, especially the gelatinases MMP- 2 and MMP-9, are increased in the lung of HPS mice.

MMP activity is increased in the lung epithelium of HPS2 mice

Because MMPs are produced by many different cell types, we next sought to localize the expression of
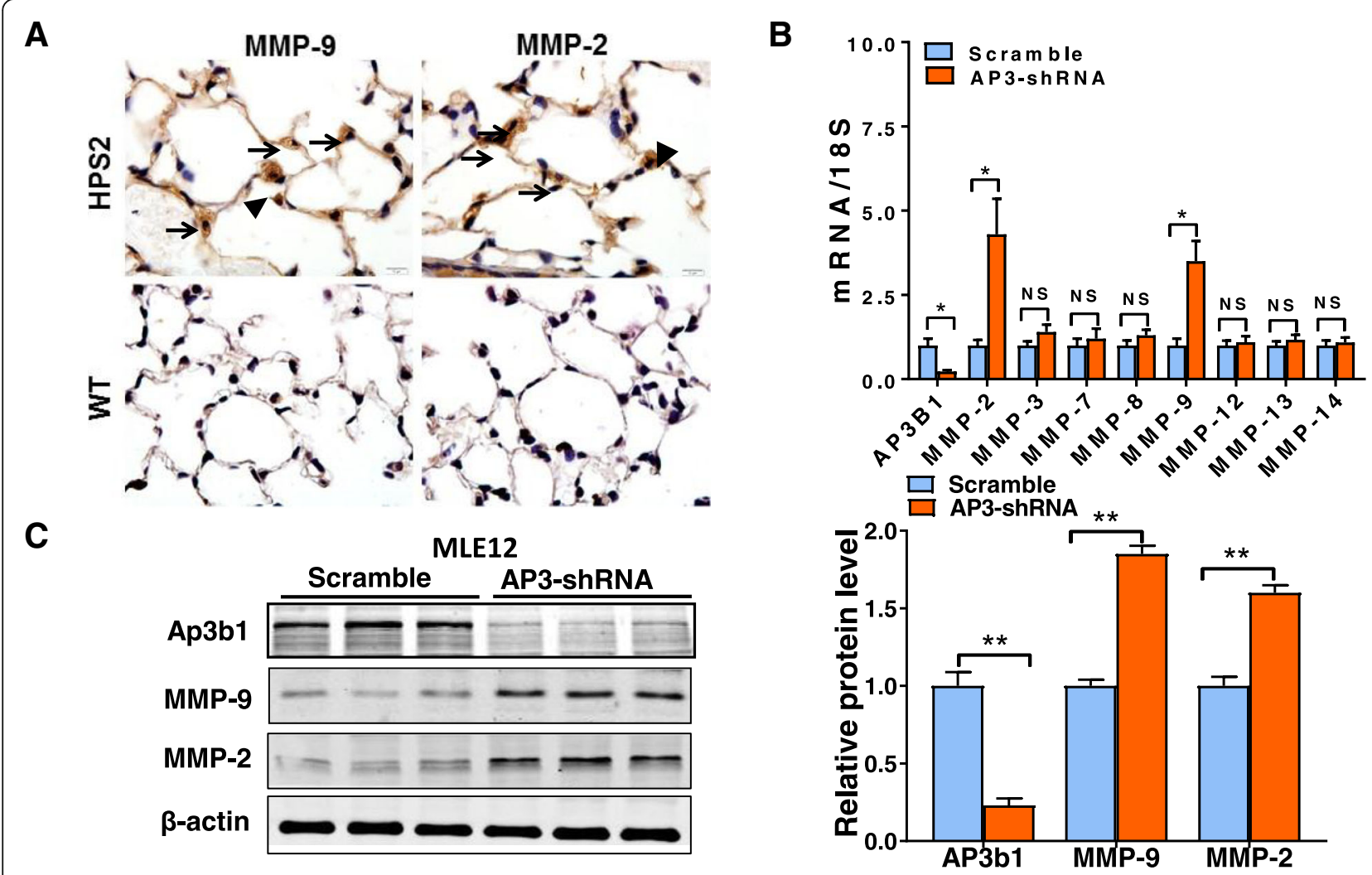

Fig. 2 HPS2 deficiency leads to increased MMP expression in lung epithelial cells. a Immunohistochemical staining for MMP-2 and MMP-9 (brown staining) in the lungs of wild-type and HPS2 mice. Staining is not detected in the lungs of wild-type mice and alveolar epithelial type II cells are difficult to visualize due to normal size lamellar bodies. Expression of MMP-2 and MMP-9 appears to be increased most significantly in alveolar epithelial type II cells, as indicated by high intensity brown staining in cells containing prominent lamellar bodies (arrows). Brown staining was also evident in some alveolar macrophages (arrow heads) and in the interstitial space. b, c shRNA knockdown of the Ap3b1 gene in MLE12 cells leads to a marked upregulation in transcript ( $n=4$, per group) and protein levels for MMP-2 and MMP-9. Immunoblot is representative of at least two different blots and densitometry analyses (bar graphs) ( $n=5$ per group, ${ }^{*} p<0.05$, HPS 2 vs. control). Data are expressed as mean \pm SE, and statistical significance was assessed using a Student's unpaired t test 
MMP-2 and -9 in the HPS2 mouse lung. As shown in Fig. 2a, immunostaining for MMP-2 and -9 did not detect significant protein expression in the lungs of wild-type mice. In contrast, we found that levels of both enzymes were readily detectable in the lungs of HPS2 mice and that staining was most abundant in AEC2 of the distal pulmonary epithelium, as judged by the cellular location and presence of lamellar bodies (vacuolated structures) in intensely stained cells (brown color) (Fig. 2a). Of note, lower intensity staining was also observed in other regions of HPS2 lung, including the pulmonary interstitium and alveolar macrophages, suggesting that mesenchymal cells might also contribute to elevated MMP levels in the lungs of these mice.

Because expression of MMP-2 and -9 was readily apparent in AEC2, we next sought to determine whether epithelial deficiency of the $A p 3 b 1$ gene could by itself increase the expression of MMP enzymes. To test this, we performed shRNA knockdown of the $A p 3 b 1$ gene in murine lung epithelial 12 (MLE12) cells, a cell line often used to model AEC2 in culture [28, 29]. Consistent with findings in vivo, we found that shRNA knockdown of $A p 3 b 1$ readily increased MMP-2 and -9 expression, as demonstrated by a greater than 2-fold increase in transcript levels and a nearly $50 \%$ increase in protein levels for both MMP enzymes (Fig. 2b, c).

\section{Matrix metalloproteinase activity is increased in the lung of HPS2 mice after bleomycin}

Expression of MMPs is known to increase in response to pro-fibrotic pulmonary insults, leading us to examine whether levels of these enzymes were further dysregulated in the HPS2 lung after pulmonary challenge. To test this, we administered a one-time, low dose $(0.025 \mathrm{U})$ of bleomycin into the oropharynx of wild-type and HPS2 mice. The decision to use a low-dose of bleomycin was based on the understanding that HPS mice are exquisitely sensitive to this genotoxic insult, and that higher doses are universally fatal $[17,18,26]$. Consistent with this being a mild pulmonary insult, we found that low-dose bleomycin had little to no effect on the expression of MMPs in the lungs of wild-type mice at day 7 after injury (data not shown). In contrast, transcript levels for all of the MMPs assessed were significantly increased in the lungs of HPS2 mice relative to injured wild-type controls (Fig. 3a). Moreover, elevated transcript

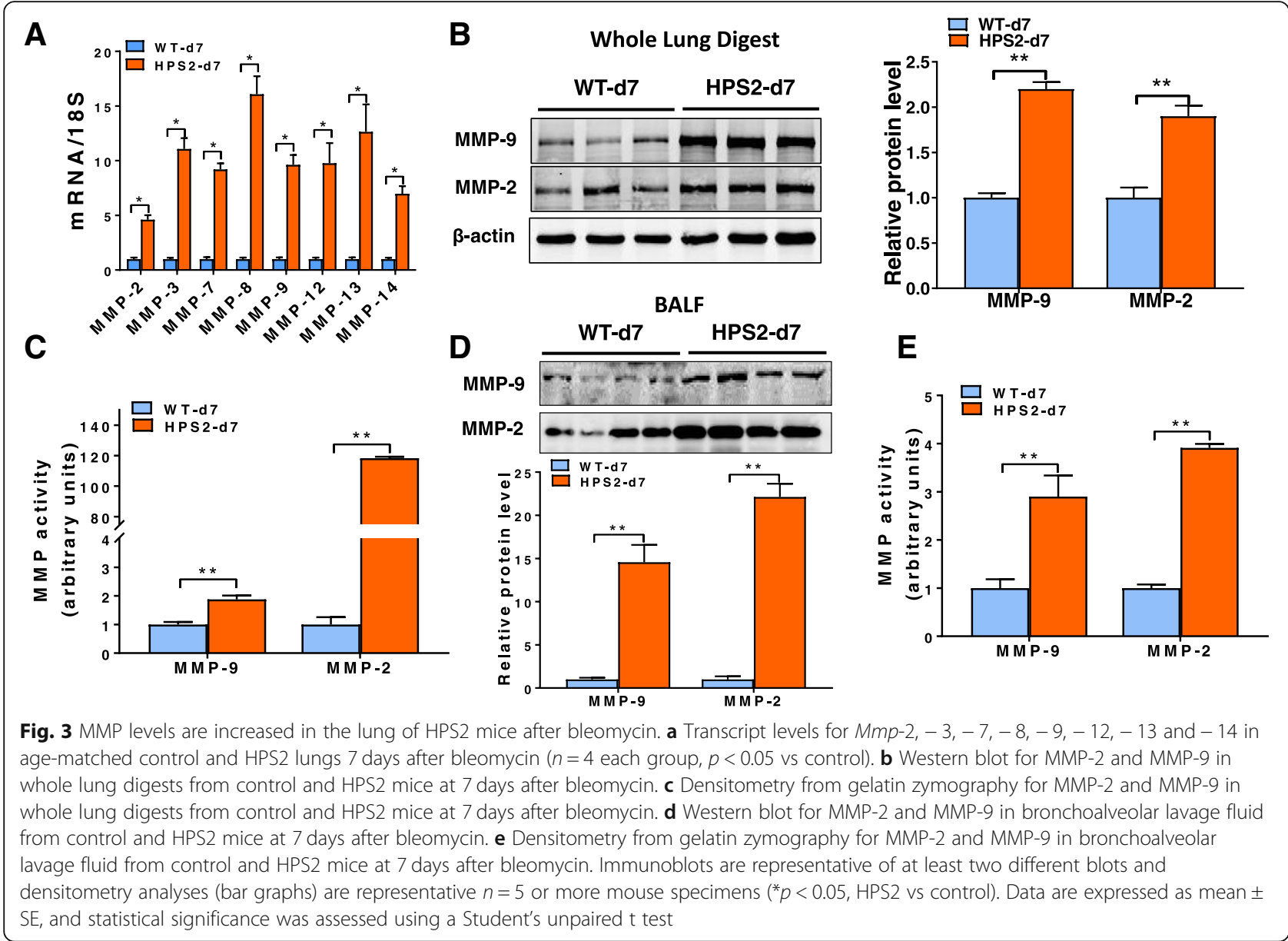


levels were also associated a marked upregulation in protein expression (Fig. 3b, d) and a dramatic increase in enzymatic activity for MMP-2 and -9 in whole lung lysates and BAL fluid (Fig. 3c, e).

\section{Elevated MMP levels are associated with an increase in Akt activation in HPS}

Since broadly inhibiting MMP activity has been associated with significant toxicity in numerous cancer studies [19], we sought to examine the effects of inhibiting an upstream regulator of MMP activity. This would also circumvent the need to simultaneously inhibit multiple MMP enzymes. Recent work has shown that MMP expression can be regulated by the enzyme Akt [21, 31, 32], leading us to hypothesize that dysregulation of Akt might contribute to altering MMP expression in the HPS lung. To test this hypothesis, we compared levels of total and phosphorylated forms of this enzyme in control and HPS tissues. Although we did not detect a significant increase in total Akt levels, the activated form of this enzyme was dramatically increased in whole lung digests of HPS2 mice at baseline and at 7 days after bleomycin. Similarly to whole lung tissues, we found that phosphorylated Akt levels were also increased in AP3 deficient lung epithelial cells (Fig. 4b) at baseline and at $24 \mathrm{~h}$ after bleomycin exposure (Fig. 4c) and that this associated with an upregulation in MMP-2 and -9 expression (Fig. 4d). In order to determine whether Akt regulates MMP expression, we exposed cells to a pharmacological inhibitor of Akt to examine the effects on MMP levels. As shown in Fig. 4e, we found that pharmacological inhibition of Akt significantly reduced MMP levels in bleomycin-exposed cells, supporting the notion that chronic activation of Akt contributes to elevated MMP expression in the HPS lung.

\section{MMP activity is increased in the lungs of HPS1 mice}

Next, to determine whether MMP expression is dysregulated in other HPS models, we measured transcript levels for various MMPs in the lungs of HPS1 mice. Strikingly, we detected a marked upregulation in transcript levels for multiple MMPs in the lungs of HPS1 mice, including Mmp-2 and -9 as well as the Mmps 3, $8,-12$ and -14 (Fig. 5a). Similarly to HPS2 mice, we also found that protein levels for MMP-2 and -9 were increased in whole lung digests and that MMP-2 gelatinase activity was increased in the BALF fluid relative to age-matched controls (Fig. 5b, c). Likewise, transcript

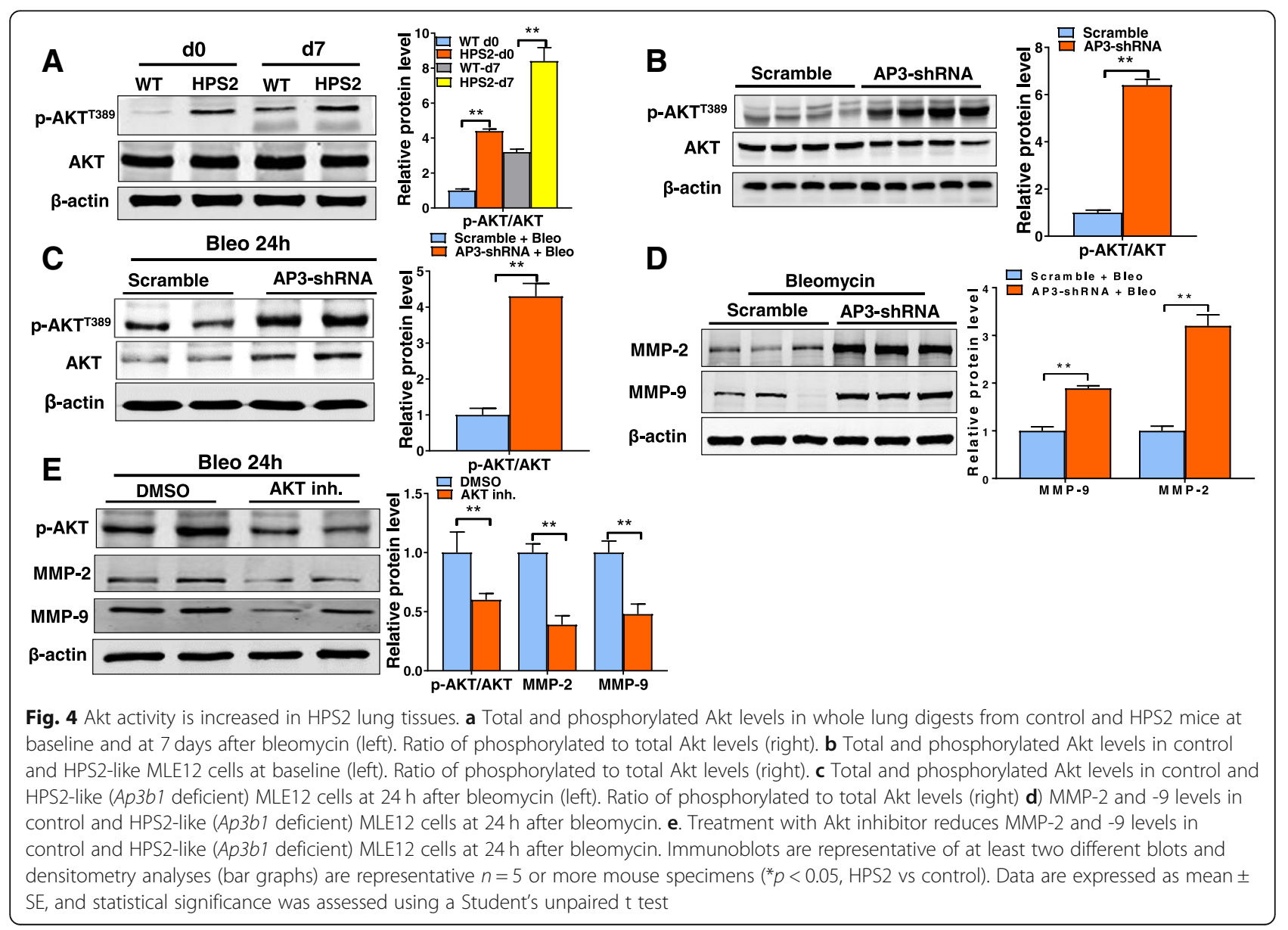



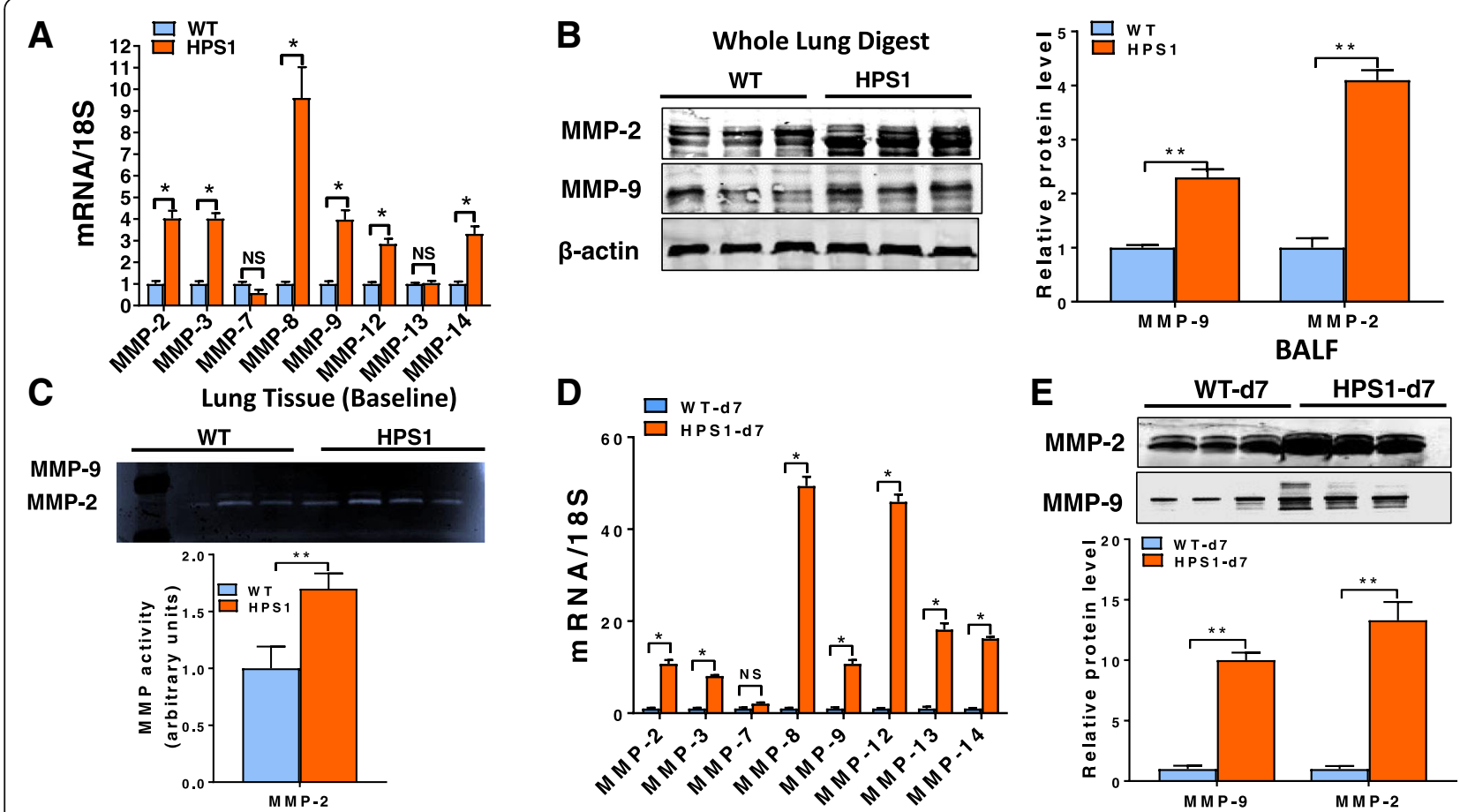

Fig. 5 MMP levels are increased in the lungs of HPS1 mice. a Transcript levels for Mmp-2, $-3,-8,-9,-12,-13$ and -14 in age-matched control and HPS1 lungs at baseline ( $n=4$ each group, $p<0.05$ vs control). b Western blot (left) for MMP- 2 and MMP-9 in whole lung digests from control and HPS1 mice at baseline. c Gelatin zymography for MMP-2 and MMP-9 in lungs of control and HPS1 mice (top). Activity of MMP-2 but not MMP-9 was significantly increased in the lungs of HPS1 mice. $\mathbf{d}$ Transcript levels for Mmp-2, $-3,-7,-8,-9,-12,-13$ and -14 in age-matched control and HPS1 lungs 7 days after bleomycin ( $n=4$ each group, $p<0.05$ vs control). e Western blot for MMP-2 and MMP-9 in whole lung digests from control and HPS1 mice at 7 days after bleomycin. Immunoblots are representative of at least two different blots and densitometry analyses (bar graphs) are representative of $n=5$ or more mouse specimens ( ${ }^{*} p<0.05$, HPS2 vs control). Data are expressed as mean \pm SE, and statistical significance was assessed using a Student's unpaired t test or multiple comparisons

levels for MMPs were also dramatically increased in the lungs of HPS1 mice after bleomycin (Fig. 5d), and this associated with elevated MMP-2 and -9 protein levels in whole lung tissue digests (data not shown) and BALF (Fig. 5e).

\section{MMP activity is increased in the lung of HPS patients}

Finally, to determine whether findings in mouse models were relevant to human disease, we assessed whether levels or activity of MMPs were altered in the lungs of HPS patients. As shown in Fig. 6, we found that protein levels for both MMP-2 and -9 were significantly increased in BALF of HPS patients relative to controls. Moreover, this associated with a significant upregulation in MMP-2 activity (Fig. 7a), although MMP-9 activity did not significantly differ between control and HPS patients (Fig. 7b). Interestingly, neither levels of MMP-2 and -9 nor activity of MMP-2 associated with the presence or absence of fibrosis or measures of lung function, such as diffusing for carbon monoxide or forced vital capacity (Fig. 7c, d).

\section{Discussion}

Mutations linked to HPS have been well-characterized but how these mutations ultimately lead to the development of pulmonary fibrosis remains unknown. In this study, we demonstrated that mutations in two different HPS genes lead to a similar upregulation in the expression and activity of MMPs in the mouse lung. Furthermore, we found that these changes occurred before the onset of lung fibrosis and were magnified after instillation of a low-dose of bleomycin into the lung. Additionally, we uncovered that increased MMP levels were also observed in the BALF of subjects with HPS, and that these changes, like in mice, were detectable in some individuals with no evidence of lung disease. Taken together, these findings indicate that HPS-related genes are important for regulating MMPs in the lung, and suggest that altered MMP expression due to HPS mutations might contribute to lung disrepair and fibrotic remodeling.

A large number of MMP genes exist within the mouse and human genome [7]. For example, at least 23 different MMP genes have been identified within the mouse 


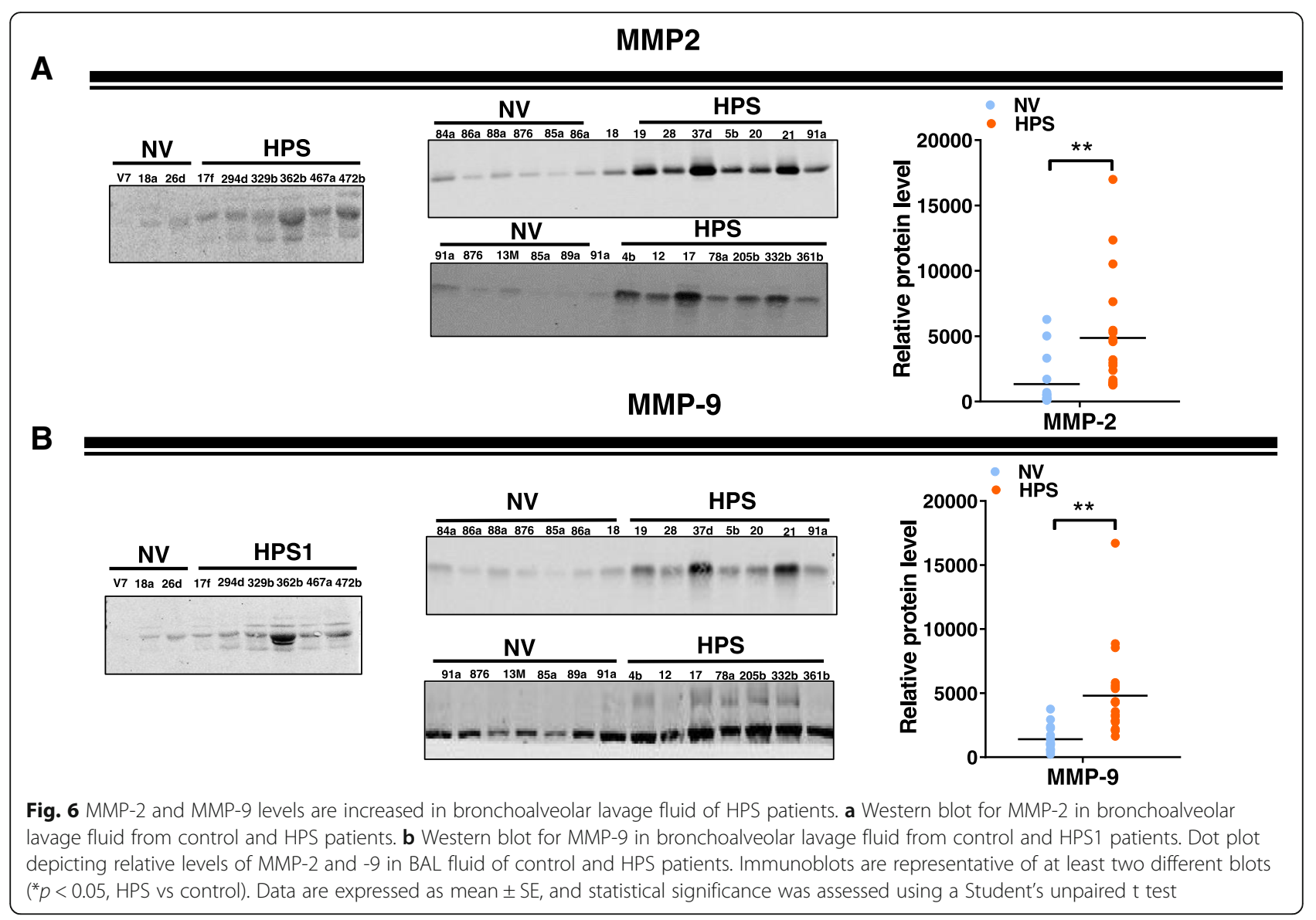

genome and even more are believed to exist in humans. In this study, we used a targeted approach to examine MMP levels in the lung, measuring only those enzymes known to be expressed in mouse or human respiratory tissues [7]. Using this targeted approach, we uncovered the upregulation of multiple MMPs in the lungs of HPS mice. Interestingly, although transcript levels for MMP-2 and -9 were most significantly increased in HPS2 mice, we detected a broad upregulation in many different MMP transcripts in the lungs of HPS1 mice, including a greater than 2-fold increase in levels for MMP-2, - 3, $8,-9,-12$ and -14 . These findings suggest the intriguing possibility that HPS is a heterogeneous group of disorders, and that pathological processes contributing to the development of pulmonary fibrosis might differ among individuals carrying different HPS mutations.

Although MMPs are produced by many different cell types in the lung, our findings suggest that epithelial cells are an important source of MMP production in the HPS lung. This was demonstrated by the marked increase in MMP-2 and -9 expression in the distal pulmonary epithelium of HPS mice and by the striking increase in MMP expression in cultured lung epithelial cells after knocking down the $A p 3 b 1$ gene. Importantly, these findings support the current paradigm in both the IPF and HPS fields that epithelial dysfunction and abnormal crosstalk of epithelial cells with mesenchymal cells contributes to the development of disease [26, 33-36].

Akt is emerging as an important pathogenic player in pulmonary fibrosis $[20,21]$. For example, Akt activation has been tightly linked to growth, survival and differentiation of activated lung fibroblasts and inhibition of this enzyme has been shown to reduce experimentallyinduced pulmonary fibrosis in mice [20, 31, 32, 37]. In addition, Akt activity is also known to be increased in the lung epithelium of mice with pulmonary fibrosis as well as the lung epithelium of patients with IPF [21]. In epithelial cells, chronic activation of Akt is thought to prime the lung for injury through reducing the expression of cell-cell junctional proteins and impairing epithelial barrier protection. Consistent with this, our study provides further support for the concept that hyperactivation of Akt contributes to the development of pulmonary fibrosis in HPS and that targeting this kinase might be a strategy for preventing or treating this disease.

Classically, levels of MMP enzymes are relatively low in healthy tissues and dramatically increase in response 


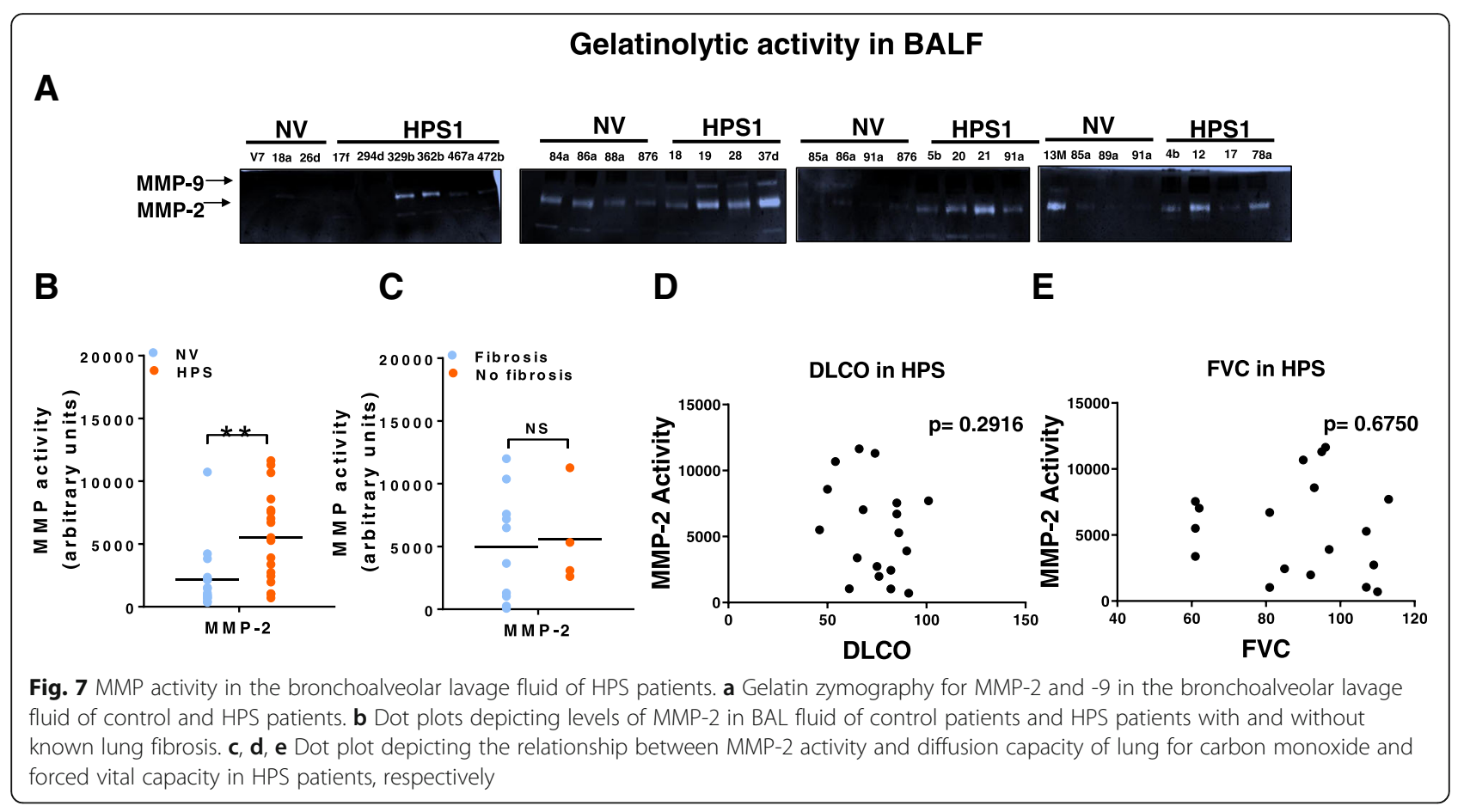

to tissue insults or disease states $[6,7,10,38]$. However, to our surprise, we found that levels of MMP enzymes were markedly increased in the HPS lung under homeostatic conditions. This included the lungs of HPS1 and HPS2 mice as well as the lungs of HPS subjects without evidence of disease. Interestingly, we did not observe a relationship between MMP activity and lung function (diffusion capacity or forced vital capacity) in our cohort of HPS patients, suggesting the intriguing possibility that chronically elevated MMP levels might contribute to the onset rather than the progression of disease. In this scenario, we wonder whether elevated MMP levels might either prime the lung for injury or make it susceptible to disrepair. Importantly, our study investigated only a limited number of MMPs, leaving the possibility that associations might be identified between other MMPs and disease activity.

Our study has several notable limitations. First, we focused only on HPS mouse models known to be susceptible to pulmonary fibrosis, preventing us from determining whether MMP levels are also dysregulated in the lungs of mice harboring other HPS mutations. Second, we measured activity of only MMP-2 and -9 in our samples, which does not allow us to comment on whether activity of other MMPs are elevated in the HPS lung. Third, our study measured MMP levels in a small cohort of patients and our population included a mixture of different HPS populations, including two individuals with HPS4 mutations. Finally, although bleomycin is considered the gold-standard pulmonary fibrosis model, it has significant limitations, including the fact that inflammation drives much of the fibrotic remodeling and tissue remodeling is completely reversible to this insult. Despite these shortcomings, the observation that similar MMPs are dysregulated in both the HPS mouse and human lung supports the validity of using this model in our investigations.

\section{Conclusions}

In summary, our findings indicate that MMP levels are upregulated in the HPS lung and that these changes precede the development of pulmonary fibrosis. Future studies determining whether elevated MMP levels assist in the diagnosis of HPS will be important. Furthermore, understanding whether dysregulation of MMPs contributes to the onset or progression of HPS lung disease will ultimately be important for advancing understanding of disease and laying the foundation for new and more effective treatments.

\section{Additional file}

Additional file 1: Clinical Demographics of HPS and control subjects. (PDF $133 \mathrm{~kb}$ )

\section{Abbreviations}

AKT: Protein Kinase B; Ap3b1: Adaptor protein complex 3-subunit beta-1; BALF: Bronchoalvelolar lavage fluid; Bloc3: Biogenesis of lysosome-related organelles complexes 3; HPS: Hermansky-Pudlak Syndrome; IPF: Idiopathic Pulmonary Fibrosis; MMP: Matrix Metalloproteinase; PI3K: Phosphatidylinositol 3-kinase 


\section{Acknowledgements}

Not applicable.

\section{Authors' contributions}

Data analysis and interpretation, RS, RK, CN, JR, BRC, FR Writing, RS FR Execution of experiments, RK, DS, KCM, DS, RP Planning. RK, DS, FR Funding, RS and FR Acquisition of data: RK, DS, KCM, DS, RP. All authors participated in the intellectual revision and approved the final version of the manuscript.

\section{Funding}

NIH R01 HL131784 (RS) and NIH R01HL136833 (F.R.)

\section{Availability of data and materials}

The datasets used and/or analyzed during the current study are available from the corresponding author on reasonable request.

\section{Ethics approval and consent to participate}

All patients provided written informed consent to protocols 95-HG-0193 (clinicaltrials.gov NCT00001456) and 04-HG-0211 (clinicaltrials.gov NCT00084305). All study protocols were approved by the Institutional Review Boards at Thomas Jefferson University and the National Human Genome Research Institute prior to the initiation of any studie.

\section{Consent for publication}

All the co-authors have read the manuscript and approved its submission to "Orphanet Journal of Rare Disease".

\section{Competing interests}

The authors declare that they have no competing interests.

\section{Author details}

${ }^{1}$ Center for Translational Medicine and Jane and Leonard Korman Lung Center, Thomas Jefferson University, Philadelphia, USA. ${ }^{2}$ Medical Genetics Branch, National Human Genome Research Institute, National Institutes of Health, Bethesda, MD, USA. ${ }^{3}$ Center for Translational Medicine and Jane and Leonard Korman Respiratory Institute, Thomas Jefferson University, 1020 Locust Street, JAH 354, Philadelphia, PA 19107, USA.

Received: 5 March 2019 Accepted: 25 June 2019

Published online: 04 July 2019

\section{References}

1. Erickson RP. Pigment, platelets, and Hermansky-Pudlak in human and mouse. Proc Natl Acad Sci U S A. 1997;94(17):8924-5

2. Swank RT, Novak EK, McGarry MP, Rusiniak ME, Feng L. Mouse models of Hermansky Pudlak syndrome: a review. Pigment Cell Res. 1998;11(2):60-80.

3. Anikster $Y$, Huizing M, White J, Shevchenko YO, Fitzpatrick DL, Touchman JW, Compton JG, Bale SJ, Swank RT, Gahl WA, et al. Mutation of a new gene causes a unique form of Hermansky-Pudlak syndrome in a genetic isolate of Central Puerto Rico. Nat Genet. 2001;28(4):376-80.

4. Vicary GW, Vergne $Y$, Santiago-Cornier A, Young LR, Roman J. Pulmonary fibrosis in Hermansky-Pudlak syndrome. Ann Am Thorac Soc. 2016;13(10): 1839-46.

5. Mohammed M, Al-Hashmi N, Al-Rashdi S, Al-Sukaiti N, Al-Adawi K, Al-Riyami M, Al-Maawali A. Biallelic mutations in AP3D1 cause Hermansky-Pudlak syndrome type 10 associated with immunodeficiency and seizure disorder. Eur J Med Genet. 2018

6. Greenlee KJ, Werb Z, Kheradmand F. Matrix metalloproteinases in lung: multiple, multifarious, and multifaceted. Physiol Rev. 2007;87(1):69-98.

7. Parks WC, Shapiro SD. Matrix metalloproteinases in lung biology. Respir Res. 2001;2(1):10-9

8. Cataldo D, Munaut C, Noel A, Frankenne F, Bartsch P, Foidart JM, Louis R MMP-2- and MMP-9-linked gelatinolytic activity in the sputum from patients with asthma and chronic obstructive pulmonary disease. Int Arch Allergy Immunol. 2000;123(3):259-67.

9. Chelladurai P, Seeger W, Pullamsetti SS. Matrix metalloproteinases and their inhibitors in pulmonary hypertension. Eur Respir J. 2012:40(3):766-82.

10. Craig VJ, Zhang L, Hagood JS, Owen CA. Matrix metalloproteinases as therapeutic targets for idiopathic pulmonary fibrosis. Am J Respir Cell Mol Biol. 2015;53(5):585-600.
11. Henry MT, McMahon K, Mackarel AJ, Prikk K, Sorsa T, Maisi P, Sepper R, Fitzgerald MX, O'Connor CM. Matrix metalloproteinases and tissue inhibitor of metalloproteinase-1 in sarcoidosis and IPF. Eur Respir J. 2002:20(5):1220-7.

12. Chen J, Doyle TJ, Liu Y, Aggarwal R, Wang X, Shi Y, Ge SX, Huang H, Lin Q, Liu W, et al. Biomarkers of rheumatoid arthritis-associated interstitial lung disease. Arthritis Rheumatol. 2015;67(1):28-38.

13. Kim WU, Min SY, Cho ML, Hong KH, Shin YJ, Park SH, Cho CS. Elevated matrix metalloproteinase-9 in patients with systemic sclerosis. Arthritis Res Ther. 2005;7(1):R71-9.

14. Cabrera S, Gaxiola M, Arreola UL, Ramirez R, Jara P, D'Armiento J, Richards T, Selman M, Pardo A. Overexpression of MMP9 in macrophages attenuates pulmonary fibrosis induced by bleomycin. Int J Biochem Cell Biol. 2007: 39(12):2324-38

15. Craig VJ, Quintero PA, Fyfe SE, Patel AS, Knolle MD, Kobzik L, Owen CA. Profibrotic activities for matrix metalloproteinase-8 during bleomycinmediated lung injury. J Immunol. 2013;190(8):4283-96.

16. Garcia-Prieto E, Gonzalez-Lopez A, Cabrera S, Astudillo A, GutierrezFernandez A, Fanjul-Fernandez M, Batalla-Solis E, Puente XS, Fueyo A, Lopez-Otin C, et al. Resistance to bleomycin-induced lung fibrosis in MMP-8 deficient mice is mediated by interleukin-10. PLoS One. 2010;5(10):e13242.

17. Lyerla TA, Rusiniak ME, Borchers M, Jahreis G, Tan J, Ohtake P, Novak EK, Swank RT. Aberrant lung structure, composition, and function in a murine model of Hermansky-Pudlak syndrome. Am J Physiol Lung Cell Mol Physiol. 2003;285(3):L643-53.

18. McGarry MP, Reddington M, Novak EK, Swank RT. Survival and lung pathology of mouse models of Hermansky-Pudlak syndrome and ChediakHigashi syndrome. Proc Soc Exp Biol Med. 1999;220(3):162-8.

19. Coussens LM, Fingleton B, Matrisian LM. Matrix metalloproteinase inhibitors and cancer: trials and tribulations. Science. 2002;295(5564):2387-92.

20. Horowitz JC, Rogers DS, Sharma V, Vittal R, White ES, Cui Z, Thannickal VJ. Combinatorial activation of FAK and AKT by transforming growth factorbeta1 confers an anoikis-resistant phenotype to myofibroblasts. Cell Signal. 2007:19(4):761-71.

21. Miyoshi K, Yanagi S, Kawahara K, Nishio M, Tsubouchi H, Imazu Y, Koshida R, Matsumoto N, Taguchi A, Yamashita S, et al. Epithelial Pten controls acute lung injury and fibrosis by regulating alveolar epithelial cell integrity. Am J Respir Crit Care Med. 2013;187(3):262-75.

22. El-Chemaly S, Young LR. Hermansky-Pudlak Syndrome. Clin Chest Med. 2016:37(3):505-11.

23. Seward SL Jr, Gahl WA. Hermansky-Pudlak syndrome: health care throughout life. Pediatrics. 2013;132(1):153-60.

24. Ren P, Rosas IO, Macdonald SD, Wu HP, Billings EM, Gochuico BR. Impairment of alveolar macrophage transcription in idiopathic pulmonary fibrosis. Am J Respir Crit Care Med. 2007:175(11):1151-7.

25. Tang $X$, Yamanaka S, Miyagi Y, Nagashima Y, Nakatani Y. Lung pathology of pale ear mouse (model of Hermansky-Pudlak syndrome 1) and beige mouse (model of Chediak-Higashi syndrome): severity of giant lamellar body degeneration of type II pneumocytes correlates with interstitial inflammation. Pathol Int. 2005;55(3):137-43.

26. Young LR, Gulleman PM, Bridges JP, Weaver TE, Deutsch GH, Blackwell TS, McCormack FX. The alveolar epithelium determines susceptibility to lung fibrosis in Hermansky-Pudlak syndrome. Am J Respir Crit Care Med. 2012; 186(10):1014-24

27. Kim JY, Choeng HC, Ahn C, Cho SH. Early and late changes of MMP-2 and MMP9 in bleomycin-induced pulmonary fibrosis. Yonsei Med J. 2009;50(1):68-77.

28. Romero F, Hong X, Shah D, Kallen CB, Rosas I, Guo Z, Schriner D, Barta J, Shaghaghi H, Hoek JB, et al. Lipid synthesis is required to resolve endoplasmic reticulum stress and limit fibrotic responses in the lung. Am J Respir Cell Mol Biol. 2018;59(2):225-36.

29. Romero F, Shah D, Duong M, Penn RB, Fessler MB, Madenspacher J, Stafstrom W, Kavuru M, Lu B, Kallen CB, et al. A pneumocyte-macrophage paracrine lipid axis drives the lung toward fibrosis. Am J Respir Cell Mol Biol. 2015:53(1):74-86.

30. Shah D, Romero F, Stafstrom W, Duong M, Summer R. Extracellular ATP mediates the late phase of neutrophil recruitment to the lung in murine models of acute lung injury. Am J Physiol Lung Cell Mol Physiol. 2014; 306(2):L152-61.

31. Cho SJ, Chae MJ, Shin BK, Kim HK, Kim A. Akt- and MAPK-mediated activation and secretion of MMP-9 into stroma in breast cancer cells upon heregulin treatment. Mol Med Rep. 2008;1(1):83-8. 
32. Kim D, Kim S, Koh H, Yoon SO, Chung AS, Cho KS, Chung J. Akt/PKB promotes cancer cell invasion via increased motility and metalloproteinase production. FASEB J. 2001;15(11):1953-62.

33. Zoz DF, Lawson WE, Blackwell TS. Idiopathic pulmonary fibrosis: a disorder of epithelial cell dysfunction. Am J Med Sci. 2011;341(6):435-8.

34. Misharin AV, Morales-Nebreda L, Reyfman PA, Cuda CM, Walter JM, McQuattie-Pimentel AC, Chen Cl, Anekalla KR, Joshi N, Williams KJN, et al. Monocyte-derived alveolar macrophages drive lung fibrosis and persist in the lung over the life span. J Exp Med. 2017;214(8):2387-404.

35. Young LR, Gulleman PM, Short CW, Tanjore H, Sherrill T, Qi A, McBride AP, Zaynagetdinov R, Benjamin JT, Lawson WE, et al. Epithelial-macrophage interactions determine pulmonary fibrosis susceptibility in HermanskyPudlak syndrome. JCI Insight. 2016;1 (17):e88947.

36. Zhou Y, He CH, Yang DS, Nguyen T, Cao Y, Kamle S, Lee CM, Gochuico BR Gahl WA, Shea BS, et al. Galectin-3 interacts with the CHI3L1 Axis and contributes to Hermansky-Pudlak syndrome lung disease. J Immunol. 2018; 200(6):2140-53.

37. Manning BD, Toker A. AKT/PKB signaling: navigating the network. Cell. 2017; 169(3):381-405.

38. Sueblinvong V, Neujahr DC, Mills ST, Roser-Page S, Ritzenthaler JD, Guidot D, Rojas M, Roman J. Predisposition for disrepair in the aged lung. Am J Med Sci. 2012:344(1):41-51.

\section{Publisher's Note}

Springer Nature remains neutral with regard to jurisdictional claims in published maps and institutional affiliations.

Ready to submit your research? Choose BMC and benefit from:

- fast, convenient online submission

- thorough peer review by experienced researchers in your field

- rapid publication on acceptance

- support for research data, including large and complex data types

- gold Open Access which fosters wider collaboration and increased citations

- maximum visibility for your research: over $100 \mathrm{M}$ website views per year

At BMC, research is always in progress.

Learn more biomedcentral.com/submissions 